\title{
Correction to: Jordan domains with a rectifiable arc in their boundary
}

\section{Vasiliki Liontou $^{1}$ (D) $\cdot$ Vassili Nestoridis $^{2}$}

Published online: 27 October 2020

(c) Fondation Carl-Herz and Springer Nature Switzerland AG 2020

\section{Correction to: Annales mathématiques du Québec https://doi.org/10.1007/s40316-019-00119-0}

There is a typo error in the name of the second author. The correct name is "Nestoridis".

Original article has been corrected.

Publisher's Note Springer Nature remains neutral with regard to jurisdictional claims in published maps and institutional affiliations.

The original article can be found online at https://doi.org/10.1007/s40316-019-00119-0.

$凶$ Vasiliki Liontou

vasiliki.liontou@mail.utoronto.com

Vassili Nestoridis

vnestor@math.uoa.gr

1 Department of Mathematics, University of Toronto, 40 St George st., Toronto, ON M5S 2E4, Canada

2 Department of Mathematics, National and Kapodistrian University of Athens, Panepistemiopolis, 15784 Athens, Greece 